\title{
Compression Analysis Using Coiflets, Haar Wavelet, and SVD Methods
}

\author{
Muhamad Aznar Abdillah ${ }^{1}$, Anton Yudhana ${ }^{2}$, Abdul Fadlil $^{3}$ \\ ${ }^{I}$ Faculty of Informatic Engineering, Department of Engineering, STMIK Muhammadiyah Paguyangan Brebes, \\ Indonesia \\ ${ }^{2,3}$ Faculty of Industrial Technology, Department of Electrical Engineering, Universitas Ahmad Dahlan, Indonesia \\ ${ }^{1}$ muhamadaznar@gmail.com, ${ }^{2}$ eyudhana@ee.uad.ac.id, ${ }^{3}$ fadlil@mti.uad.ac.id
}

\begin{abstract}
The image problem lies in the amount of storage space required, to save memory as little as possible image compression is required. The image compression technique is a technique used to represent an image by reducing the quality of the original image but still retaining the information inside. This study compares the best compression method between Coiflets, Haar wavelets, and SVD with JPG image material. The comparison process has done by calculating the compression ratio (CR), Space Saving (SS), Mean Square Error (MSE), Root Mean Square Error (RMSE), and Peak Signal to Noise Ratio (PSNR). The results obtained prove that the SVD method has the highest compression ratio of 3.25 while in the case of Space Saving (SS) the Coiflets method gives the best performance with a value of 73. Measurement in terms of MSE and RMSE is the best for the Coiflets method because it has an average value. -The smallest average among all methods is $\mathbf{0 . 0 2 3 9 5}$ and $\mathbf{0 . 1 1 1 3 8 3}$. provides the best performance in maintaining compression quality. The best PSNR based image quality assessment is the Coiflets method with the highest PSNR average of $63.02 \mathrm{~dB}$. Overall, the Coiflets, Haar wavelet, and SVD compression methods used for JPG images can reduce file size and preserve image information and quality.
\end{abstract}

Keywords: image compression, coiflets; Haar wavelets, SVD.

\section{INTRODUCTION}

The need for technology is currently developing so rapidly, for example, such as information technology, which consists of several forms such as images or photos and videos [1]. The exchange of data information is currently very often done by many people, such as exchanging information about a place. To get a picture of the place, a camera is needed to record the information so that the information can be summarized in an image or photo [2]. Image can be obtained by capturing the strength of the rays reflected by the object so that the image is an image of an object [3]. Since the discovery of a tool to capture an image in the image plane in the form of a camera, this development has made its focus not only on the camera but also on the tools used to store the image [4].

The image problem lies in the amount of storage space required, to save memory as small as possible image compression is required. Image compression technique is a technique used to represent an image by reducing the quality of the original image, but still retaining the information therein [5]. Several digital image data formats are commonly used, each of which has its advantages and disadvantages. One of them is a digital image that is very common on the internet, namely a digital image in JPG format [6].

The compression process is the measurement of data files [7]. Digital image compression is an attempt to transform the data of digital image compilation into other data, without causing significant changes. This aims to reduce the redundancy of the data contained in the image so that it is transmitted efficiently [8]. The image compression technique consists of two techniques, namely lossless compression and lossy compression [9]. Lossless compression is a technique that processes original data into a more compact form without losing information in the image [8]. In general, lossy compression can produce a compression ratio greater than lossless compression [10]. Lossy compression prioritizes very high compression ratios at the expense of some lost information but is well tolerated by eye perception [11]. Some of them are the DWT transformation and Singular Value Decomposition (SVD).

Wavelet Haar is the oldest and simplest wavelet. This method is very good to be used to represent texture and shape characteristics, Haar wavelet compression is also an efficient way of image compression but is less resistant to attacks such as blur and noise [12]. SVD is a lossy compression group image compression that has been widely used, therefore even though it has high compression results, it sacrifices some missing information in the image [13]. This method is one of the 
matrix processing techniques from the branch of linear algebra [14]. One other well-known compression is using Coiflets compression, but it has a small compression ratio [15]. Compressed image formats, in general, are GIF, PNG, JPEG, and others [7].

Previous researches include research on image compression using the fractal method, with the results that image compression can be done but only has a low compression efficiency level [16]. The novelty in this research is to use three compression techniques, namely Coiflets, Haar wavelet, and Singular Value Decomposition (SVD) with the image used with JPG extension. The results of the compression will be compared with different parameters from the previous research, namely using the compression ratio and quality values in terms of MSE and PSNR.

\section{METHOD}

\section{A. Research Flow}

The steps carried out in this study consisted of the compression steps and the performance measurement stage. Fig. 1 is the stages carried out.

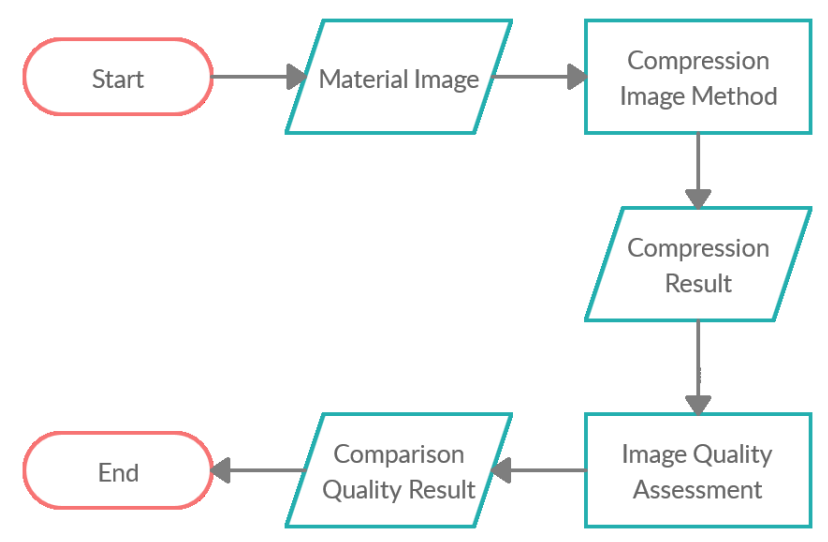

Fig. 1 Image compression stages

Fig.1.explains the first stage of preparing the materials used, namely images in JPG format, this image is used as material for the compression process. After inputting the image, the compression process is carried out using the Coiflets, Haar wavelet, and SVD methods. The next stage is the process of measuring performance using the parameters of the compression ratio calculation and image quality calculations. The stages of measuring image quality are carried out by measuring the values of Mean Squared Error (MSE), Root Mean Square Error (RMSE), and Peak Signal to Noise Ratio (PSNR). From the results of this measurement, it is then analyzed and conclusions are drawn to get the best method for compressing JPG images from the three compression methods tested.

\section{B. Compression Methods}

1) Coiflets: Discrete wavelet designed by Ingrid Daubechies, at the request of Ronald Coifman, to have a vanishing moment scaling function. This wavelet is nearly symmetrical, the wavelet function used is $\mathrm{N} / 3$ vanishing moment and the scaling function is $\mathrm{N} / 3-1$, and has been used in many applications using the Calderón-Zygmund operator [17].

2) Wavelet Haar: Haar wavelet is the oldest and simplest wavelet and this method is good to use to represent texture and shape characteristics. Besides that Haar Wavelet requires less computation time than other wavelet transforms, the features are obtained from images that have gone through the decomposition process [18][12].

3) SVD: is a mathematical method to decompose a single matrix by compressing it into three smaller matrices of the same size by reducing the data in columns and rows [13]. Each matrix $\mathrm{M}$ in the SVD, which is $\mathrm{n} \times \mathrm{n}$ in size, can be broken down into three parts as in (1) [19]:

$$
M=U \times S \times V^{T}
$$

SVD divides $M$ into three matrices: $U, S$, dan $V$. Matrix $U_{m \times n}$ satisfy $U^{T}{ }_{n \times n} \times U_{m \times n}=I_{n \times n}$. Matrix $V_{n \times n}$ satisfy $V^{T}{ }_{n \times n} \times V_{n \times n}=I_{n \times n}$, where $I_{n \times n}$ is the units matrix. The resulting $\mathrm{S}$ is a diagonal matrix, where the diagonal elements are the singular values and tell the important features of the data. When all elements are selected, SVD can reconstruct the original image without errors [20]. If the $\mathrm{k}$ element is selected, the compression efficiency is (2):

$$
\frac{n \times k+k \times k+k \times n}{n \times n}
$$

\section{Image Quality Assessment}

Objective measurements are intended to analyze image quality without human involvement. To evaluate the performance of the compression method, several measurement methods have been used in research to compare the differences between the original image and the compressed image. Measurement methods such as Compression Ratio (CR) and Space Saving (SS), Mean Square Error (MSE), Root Mean Square Error (RMSE), and Peak Signal Noise Ratio (PSNR) are used to measure the quality of the compression method on Coiflets, Haar wavelets, and SVD. 
1) Compression Ratio: Compression Ratio (CR) is a comparison between the size of the reconstructed image and the size of the original image. The higher the CR, the better the performance of the compression method [21]. Eq. 3 is used to calculate CR [22][23].

$$
C R=\frac{\text { size of original image }}{\text { size of compressed image }}
$$

2) Space Saving: to determine the effectiveness of the compression method in reducing the image size easily, the percentage of Space Saving can be obtained from (4).

$$
S S(\%)=\left(1-\frac{1}{C R}\right) \times 100
$$

3) Mean Square Error: was used to measure the degree of distortion in the reconstructed image [24]. The smaller the MSE value shows that the better image processing results, or in other words, the image after processing is closer to the original image [25]. Eq.5 is used to calculate MSE [22][26].

$$
M S E=\frac{1}{m n} \sum_{i=0}^{m-1} \sum_{j=0}^{n-1}[I(i, j)-K(i, j)]^{2}
$$

4) Root Mean Square Error: if MSE shows the cumulative square error between the compressed and original image, then RMSE is the square root of MSE. The RMSE can be obtained by the following (6).

$$
R M S E=\sqrt{M S E}
$$

5) Peak Signal Noise Ratio: the ratio between the maximum value of the measured bit depth image (8-bit

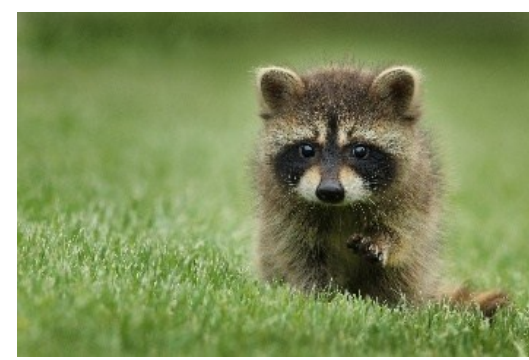

(a)

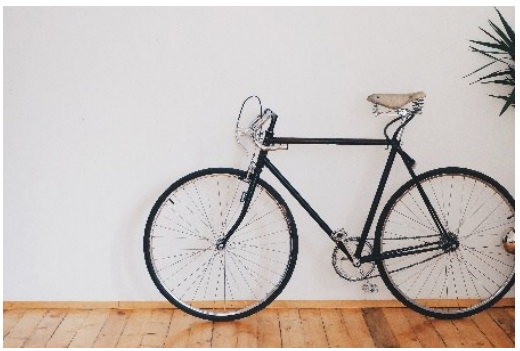

(d) image, has a maximum value of 255) and the amount of noise that affects the signal. The amount of noise is represented by the MSE (Mean Square Error) value [27]. PSNR is usually measured in decibels (dB). PSNR was used to compare the image quality before and after compression [28]. The higher the PSNR value, the better the quality of the compressed, or reconstructed image. Eq.7 is used to calculate the PSNR [29][30].

$$
P S N R=10 \log _{10}\left(\frac{R^{2}}{\sqrt{M S E}}\right)
$$

\section{RESULTS AND DISCUSSION}

The results and discussion in this section as the form of experiments on image compression has been prepared based on different methods, such as Coiflets, Wavelet Haar, and SVD. For each method, quality evaluation is carried out by comparing the compressed image and the original image based on several measurements such as Compression Ratio (CR), Space Savings (SS), Mean Squared Error (MSE), Root Mean Squared Error (RMSE) and Peak Signal to Noise. Ratio (PSNR). Fig. 2 shows four test images of different sizes that have been used for the simulation.

The material image will be an input image in compression using the Coiflets, Haar wavelet, and SVD methods. Fig. 3 below is the result of image compression performed.

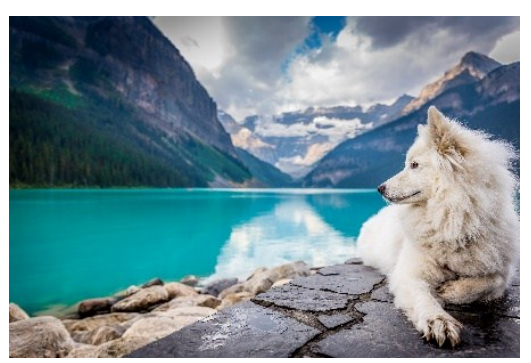

(b)

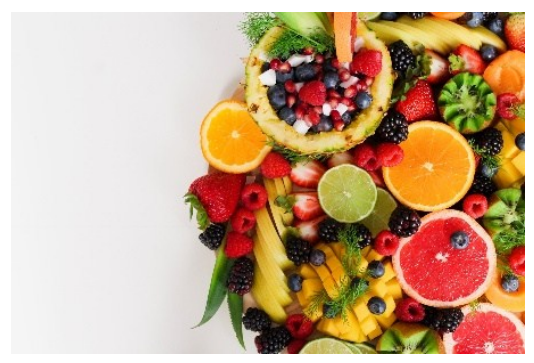

(e)

Fig. 2 Differences in the images used in the simulation, (a) racoon, (b) wolf, (c) bike, (d) fruit 


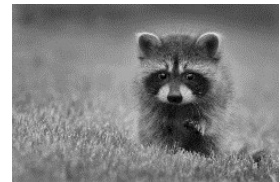

(a1)

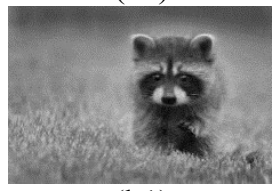

(b1)

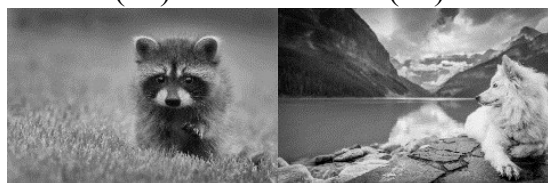

(c1)

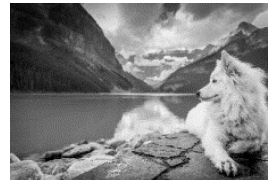

(a2)

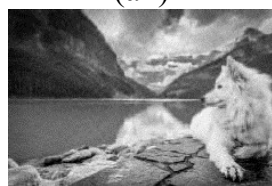

(b2)

(c2)

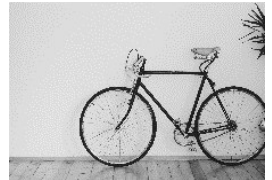

(a3)

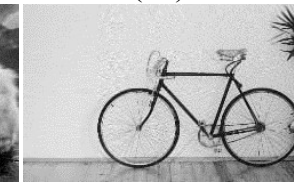

(b3)

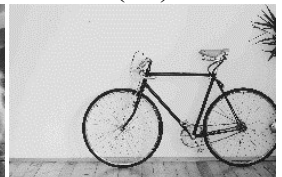

(c3)

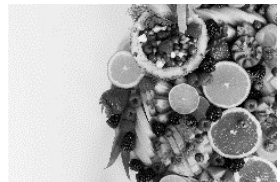

(a4)

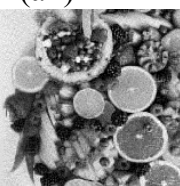

(b4)

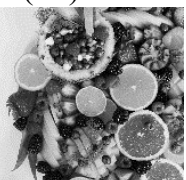

(c4)

Fig. 3 Compression image: coiflets (a) racoon (1.24), (b) wolf (1.28), (c) bike (1.38), (d) fruit (1.48); wavelet Haar (a) racoon $(1,81)$, (b) wolf $(1,69)$, (c) bike $(1,82)$, (d) fruit $(1,99)$; SVD (a) racoon $(2.99)$, (b) wolf (2.40), (c) bike (2.41), (d) fruit (5.19)

Table I shows the compression ratio (CR) and space saving (SS) of the various methods in the data. The results show that the SVD method provides the highest compression value (CR) compared to other methods. The average CR generated was 3.25 for SVD and was the highest compared to Coiflets and Haar with an average of 1.34 and 1.82. By showing the highest CR, the SVD method is expected to provide an efficient way to store and transmit other files.

In addition, it is necessary to investigate the performance of the compression method based on different measurements. Table II shows the performance of the compression method based on MSE and RMSE. The lower the MSE value, the better the compressed image quality. The experimental results show that the Coiflets method provides the lowest MSE value compared to other methods. The mean MSE values were $0.02395,8.76$, and 32.15 for the Coiflets,
Haar, and SVD methods, respectively. MSE and RMSE reveal the difference between the compressed image and the original image. A small MSE value improves image quality and reduces noise. The results of this experiment indicate that the Coiflets method is close to the original image.

Table III shows the performance of the compression method using the PSNR measurement. PSNR can be used as an indicator of the quality of perception where the PSNR which is higher than the compressed image has good quality. The results showed that Coiflets produced the highest PSNR compared to other methods with an average PSNR of $63.02 \mathrm{~dB}$. Meanwhile, the Haar and SVD methods have an average PSNR of $38.29 \mathrm{~dB}$ and $35.15 \mathrm{~dB}$. So that it can be concluded, the experimental results show that using the Coiflets method, can produce compressed images with high quality.

TABLE I

COMPARISON OF IMAGE COMPRESSION METHOD BASED ON COMPRESSION RATIO AND SPACE SAVING

\begin{tabular}{lccccccc}
\hline \multirow{2}{*}{ Image } & Original size & \multicolumn{2}{c}{ Coiflets } & \multicolumn{2}{c}{ Haar } & \multicolumn{2}{c}{ SVD } \\
\cline { 2 - 8 } & $(\mathbf{K B})$ & CR & SS (\%) & CR & SS (\%) & CR & SS (\%) \\
\hline Racoon (5472 x 3648) & 2300 & 1.24 & 78.47 & 1.81 & 44.78 & 2.99 & 66.57 \\
Wolf (5077 x 3385) & 1760 & 1.28 & 76.02 & 1.69 & 40.91 & 2.40 & 58.30 \\
Bike(6000 x 4000) & 3830 & 1.38 & 70.13 & 1.82 & 45.05 & 2.41 & 58.51 \\
Fruit (2305 x 1537) & 654 & 1.48 & 67.38 & 1.99 & 49.85 & 5.19 & 80.73 \\
\hline Average & & 1.34 & 73 & 1.82 & 45.15 & 3.25 & 66.03 \\
\hline
\end{tabular}


TABLE II

COMPARISON OF IMAGE COMPRESSION RESULTS USING MSE AND RMSE

\begin{tabular}{lcccccc}
\hline \multirow{2}{*}{ Image } & \multicolumn{2}{c}{ Coiflets } & \multicolumn{2}{c}{ Haar } & \multicolumn{2}{c}{ SVD } \\
\cline { 2 - 7 } & MSE & RMSE & MSE & RMSE & MSE & RMSE \\
\hline Racoon & 0.0007 & 0,026458 & 8.29 & 2.88 & 17.11 & 4.14 \\
Wolf & 0.087 & 0,294958 & 2.70 & 1.64 & 17.21 & 4.15 \\
Bike & 0.0058 & 0,076158 & 7.63 & 2.76 & 62.86 & 7.93 \\
Fruit & 0.0023 & 0,047958 & 16.42 & 4.05 & 31.43 & 5.61 \\
\hline Average & 0,02395 & 0,111383 & 8.76 & 2.43 & 32.15 & 5.46 \\
\hline
\end{tabular}

TABLE III

COMPARISON OF IMAGE COMPRESSION RESULTS USING PSNR

\begin{tabular}{lccc}
\hline \multirow{2}{*}{ Image } & Coiflets & Haar & SVD \\
\cline { 2 - 4 } & PSNR (dB) & PSNR (dB) & PSNR (dB) \\
\hline Racoon & 71.87 & 38.98 & 35.83 \\
Wolf & 50.94 & 43.85 & 35.8 \\
Bike & 62.71 & 34.34 & 35.8 \\
Fruit & 66.55 & 36.01 & 33.19 \\
\hline Average & 63,02 & 38.295 & 35.155 \\
\hline
\end{tabular}

The results of the experimental scenario show that the SVD method has the best performance in terms of Compression Ratio (CR) of 3.25 while in the case of Space Saving (SS) the Coiflets method gives the best performance with a value of 73. Measurement in terms of MSE and RMSE is the best. the Coiflets method, because it has the smallest average value among all methods, namely 0.02395 and 0.111383 . provides the best performance in maintaining compression quality. The best PSNR based image quality assessment is the Coiflets method with the highest PSNR average of $63.02 \mathrm{~dB}$.

\section{CONCLUSION}

The results of the research that has been done can be concluded that the three compression methods can reduce the image size. The compression methods used such as Coiflets, Haar wavelet and SVD have been tested using four JPG photos. In this test, it was found that the SVD compression method has a greater compression ratio (CR) than the other methods. The computation of the MSE, RMSE, and PSNR values that have been carried out has concluded that good compression in terms of MSE, RMSE, and PSNR values is the Coiflets method, but this method has a small compression ratio compared to SVD compression.
These results were obtained from four photos that had been tested for compression, the average compression ratio of the SVD was 3.25 for SVD and was the highest compared to Coiflets and Haar with an average of 1.34 and 1.82. By showing the highest CR, the SVD method is expected to provide an efficient way to store and transmit other files. The PSNR value obtained from the four experiments also did not decrease significantly so that the information presented did not change too much. The PSNR values of the three methods all have an average value above $30 \mathrm{~dB}$. Overall, the Coiflets, Haar wavelet, and SVD compression methods can be used for JPG images, can reduce file size, and maintain image information and quality.

\section{REFERENCES}

[1] I. Riadi, A. Yudhana, and W. Y. Sulistyo, "Image Forensics Analysis To Detect Falsification of Digital Photos," Mob. Forensics, vol. 1, no. 1, pp. 13-21, 2019.

[2] A. Muchammad and P. Diah, "Image Compression Using the Fractal Method," Universitas Muhammadiyah Surakarta, 2018.

[3] S. Saifullah, Sunardi, and A. Yudhana, "Comparative Analysis of Original Image Processing and Cropping Results for Egg Identification," J. Tek. Inform. dan Sist. Inf., vol. 2, no. 3, 2018, doi: 10.28932/jutisi.v2i3.512.

[4] G. D. Sanjaya, R. Hadi, N. Luh, and G. Pivin, 
"Kompresi Citra Digital Menggunakan Metode Discrete Cosine Transform," pp. 38-44, 2018.

[5] B. D. Raharja and P. Harsadi, "Implementasi Kompresi Citra Digital Dengan Mengatur Kualitas Citra Digital," J. Ilm. SINUS, vol. 16, no. 2, 2018, doi: 10.30646/sinus.v16i2.363.

[6] Hendri, "Kompresi Citra dari Format BMP ke Format PNG," J. Time, vol. III, no. 1, pp. 27-31, 2014.

[7] S. M. S. Hilles and M. S. Shafii, "Image Compression and Encryption Technique : Review Paper," Int. J. Data Sci. Res., vol. 1, no. 2, pp. 0-6, 2019.

[8] R. Krasmala, A. Budimansyah, and U. T. Lenggana, "Image Compression by Combining Discrete Cosine Transform (DCT) Method and Huffman Algorithm," $J$. Online Inform., vol. 2, no. 1, p. 1, 2017, doi: 10.15575/join.v2i1.79.

[9] M. Rohman and I. Anisah, "Image Compression Technique Using Vector Quantization Method Based on Fuzzy C-Means," Semin. Nas. Tek. Inform., pp. 4-8, 2013.

[10] Jatmika and T. F. Randongkir, "Implementation of the Fractal Algorithm for Image Compression with Local Search Methods," J. Inform., vol. 9, no. 1, pp. 43-50, 2013.

[11] P. N. Andono, S. T, and Muljono, Digital Image Processing. Yogyakarta: ANDI OFFSET, 2017.

[12] L. Novamizanti and A. Kurnia, "Comparison Analysis of Haar Wavelet Transform Compression with Embedded Zerotree Wavelet on Image," ELKOMIKA J. Tek. Energi Elektr. Tek. Telekomun. Tek. Elektron., vol. 3, no. 2, p. 161, 2015, doi: 10.26760/elkomika.v3i2.161.

[13] Subinarto and E. Susanto, "Medical Image Compression Using Combination Method Singular Value Decomposition (SVD) And Discrete Wavelet Transform (DWT) To Improve Storage And Transmission Efficiency," J. LINK, vol. 12, no. 2, pp. 25-30, 2016, [Online].

Available: http://ejournal.poltekkessmg.ac.id/ojs/index.php/link/art icle/viewFile/1386\%0A/445\%0A.

[14] M. T. Hidayatullah, E. Suhartono, and I. S. S. T, "Compression Arithmetic Coding Using Cs Technique And Arithmetic Compression Coding Using Cs and Combined Technique of Dct and Svd Method," $e$ Proceeding Eng., vol. 6, no. 1, pp. 503-510, 2019.

[15] I. M. B. Saputra, A. Romadhony, and Adiwijaya, "JPEG Lossless Compression Analysis with Addition of the deflate-compressed complement," 2012.

[16] S. N. Ardhytia and Lely Hiryanto, "Sequential And Parallel Fractal Compression Algorithm For Image Compression," J. Chem. Inf. Model., vol. 53, no. 9, pp. 1689-1699, 2013.

[17] Sutarno, C. Wajah, F. I. Komputer, and U. Sriwijaya,
"Wavelet Transform Comparative Analysis," J. Generic, vol. 5, no. 2, pp. 15-21, 2010, doi: 10.1111/14710528.12638 .

[18] S. Tamboli and T. Ashta, "Image Compression Using Haar Wavelet Transform," Int. J. Adv. Res. Comput. Commun. Eng., vol. 2, no. 8, pp. 3166-3170, 2013.

[19] C. Yu, H. Li, and X. Wang, "SVD-based image compression, encryption, and identity authentication algorithm on cloud," IET Image Process, vol. 13, no. 12, pp. 2224-2232, 2019, doi: 10.1049/iet-ipr.2018.5912.

[20] K. El Asnaoui, "Image Compression Based on Block SVD Power Method," J. Intell. Syst., vol. 29, no. 1, pp. 1345-1359, 2020, doi: 10.1515/jisys-2018-0034.

[21] C. Priya, C. Ramya, R. V. Agashthiya, R. Hema, G. Mythily, and V. P. Preethi, "An Efficient Method for Secure Image Compression," Int. J. Innov. Technol. Explor. Eng., vol. 8, no. 6, pp. 266-270, 2019.

[22] P. Kumar and A. Parmar, "Versatile Approaches for Medical Image Compression: A Review V," Procedia Comput. Sci., vol. 167, pp. 1380-1389, 2020, doi: 10.1016/j.procs.2020.03.349.

[23] R. Kumar, U. Patbhaje, and A. Kumar, "An efficient technique for image compression and quality retrieval using matrix completion," J. King Saud Univ. - Comput. Inf. Sci., 2019, doi: 10.1016/j.jksuci.2019.08.002.

[24] D. C. R. Dudhagara, "An Analysis and Study of HAAR Wavelet Based Method for 2D Image Compression," Int. J. Trend Sci. Res. Dev., vol. 1, no. 5, pp. 238-242, 2017, doi: $10.31142 /$ ijtsrd2267.

[25] H. R. Fajrin, "Perbandingan Metode untuk Perbaikan Kualitas Citra Mammogram," J. SIMETRIS, vol. 7, no. 2, pp. 657-664, 2016.

[26] A. Alkholidi, A. Alfalou, and H. Hamam, "A new approach for optical colored image compression using the JPEG standards," vol. 87, pp. 569-583, 2007, doi: 10.1016/j.sigpro.2006.06.011.

[27] R. Kaur and P. Choudhary, "A Review of Image Compression Techniques," Int. J. Comput. Appl., vol. 142, no. 1, pp. 8-11, 2016, doi: 10.5120/ijca2016909658.

[28] A. S. Ahmed, "Comparative Study Among Sobel, Prewitt and Canny Edge Detection Operators Used in Image Processing," J. Theor. Appl. Inf. Technol., vol. 96, no. 19, pp. 6517-6525, 2018.

[29] I. Riadi, A. Yudhana, and W. Y. Sulistyo, "Image Quality Value Comparison Analysis on Edge Detection Method," Rekayasa Sist. dan Teknol. Inf., vol. 4, no. 2, pp. 345-351, 2020.

[30] C. Chang, Y. Chou, and J. Shen, "Improving Image Quality for JPEG Compression," Knowledge-Based Intell. Inf. Eng. Syst., vol. 3683, pp. 442-448, 2005. 